EPJ Web of Conferences 81, 01013 (2014)

DOI: $10.1051 /$ epjconf/ 20148101013

(C) Owned by the authors, published by EDP Sciences, 2014

\title{
Latest results from KLOE-2
}

\author{
Caterina Bloise ${ }^{1, a}$ \\ on behalf of the KLOE-2 Collaboration \\ ${ }^{1}$ Laboratori Nazionali di Frascati dell'INFN, Frascati, Italy.
}

\begin{abstract}
The KLOE experiment at the Frascati $\phi$-factory recently obtained results on i) CPT and Lorentz invariance tests from the study of quantum interference of the neutral kaon pairs; ii) precision measurement of the branching fraction, $\mathrm{BR}\left(\mathrm{K}^{+} \rightarrow\right.$ $\left.\pi^{+} \pi^{-} \pi^{+}(\gamma)\right)=0.05565 \pm 0.00031_{\text {stat }} \pm 0.00025_{\text {syst }}$, and iii) on dark photon searches with the analysis of the $e^{+} e^{-} \rightarrow \mu \mu \gamma$ final state. We have also studied the transition form factors of the $\phi$ meson to the pseudoscalars, $\pi^{0}$ and $\eta$, that is presented in a separate contribution to this volume.
\end{abstract}

\section{The KLOE experiment}

The KLOE experiment at the Frascati $\phi$-factory took most of the data in 2004-2006, with $2.5 \mathrm{fb}^{-1}$ of integrated luminosity at the $\phi$ peak, and about $250 \mathrm{pb}^{-1}$ at $1 \mathrm{GeV}, 20 \mathrm{MeV}$ below the resonance, for the study of di-pion and di-lepton production, and $\gamma-\gamma$ interactions. In year 2013 we successfully completed the installation of the detector upgrades, and the $D A \Phi N E$ accelerator underwent a massive renovation to improve instantaneous luminosity and operation reliability.

The KLOE detector consists of a large cylindrical Drift Chamber (DC), surrounded by a leadscintillating fiber electromagnetic calorimeter (EMC), all embedded inside a superconducting coil, providing a $0.52 \mathrm{~T}$ axial field. The drift chamber [1], $4 \mathrm{~m}$ in diameter and $3.3 \mathrm{~m}$ long, has 12,582 all-stereo tungsten sense wires with a shell made of carbon fiber-epoxy composite. The momentum resolution is $\sigma\left(p_{\perp}\right) / p_{\perp} \sim 0.4 \%$. The calorimeter [2] covers $98 \%$ of the solid angle. Each cell is read out at both ends by photomultipliers, both in amplitude and time. Energy and time resolutions are $\sigma_{E} / E=5.7 \% / \sqrt{E(\mathrm{GeV})}$ and $\sigma_{t}=57 \mathrm{ps} / \sqrt{E(\mathrm{GeV})} \oplus 100 \mathrm{ps}$.

The detector has been upgraded i) to improve vertex reconstruction near the beam interaction region (IR), ii) to increase the acceptance for low polar angle photons, and iii) to reconstruct particles passing through the $D A \Phi N E$ final focusing region. A cylindrical tracking chamber based on the Gaseous Electron Multiplier (GEM) technology [3], the first cylindrical 3-GEM detector ever built, has been installed between the beam pipe and the big Drift Chamber to track particles closer to their origin; two small stations of LYSO calorimeters [4] have been placed on the beam pipe for the detection of low polar angle photons; the final focusing region has been instrumented with sampling calorimeters done by five layers of tungsten interleaved with scintillator tiles coupled to fibers that are readout on one side by silicon photomultipliers [5]. A new data taking campaign aiming to collect $O(10) \mathrm{fb}^{-1}$ in $2-3$ years of data taking is planned to extend the experimental program in $\mathrm{kaon} / \mathrm{hadron}$ physics and on dark photon searches [6].

\footnotetext{
a e-mail: Caterina.Bloise@lnf.infn.it
} 


\section{CPT and Lorentz-invariance tests}

CPT and Lorentz invariance tests are considered a probe for physics at the Planck scale, where natural mechanisms of CPT violation are expected in connection with modifications in the space-time structure relating to quantum theory of the gravity. Space-time modifications could naturally lead to Lorentz-invariance breaking that, in the low-energy regime accessible to experiments, are described by effective field theories, such as the Standard Model Extension, SME, widely used in CPT tests from a broad class of physics sectors, from atomic to particle physics, to cosmology. In year $2014 \mathrm{KLOE}$ obtained the best sensitivity ever reached in the quark sector on CPT and Lorentz invariance, based on $1.7 \mathrm{fb}^{-1}$ of integrated luminosity. The test was performed on the entangled neutral kaon pairs, in the $\phi \rightarrow K_{S} K_{L} \rightarrow \pi^{+} \pi^{-} \pi^{+} \pi^{-}$final state, studying the interference pattern as a function of sidereal time and particle direction in celestial coordinates (fig. 1). Due to the fully-destructive quantum interference at $\Delta \tau=0$, the distribution is very sensitive to CPT-violating effects, especially for decays near the IR.

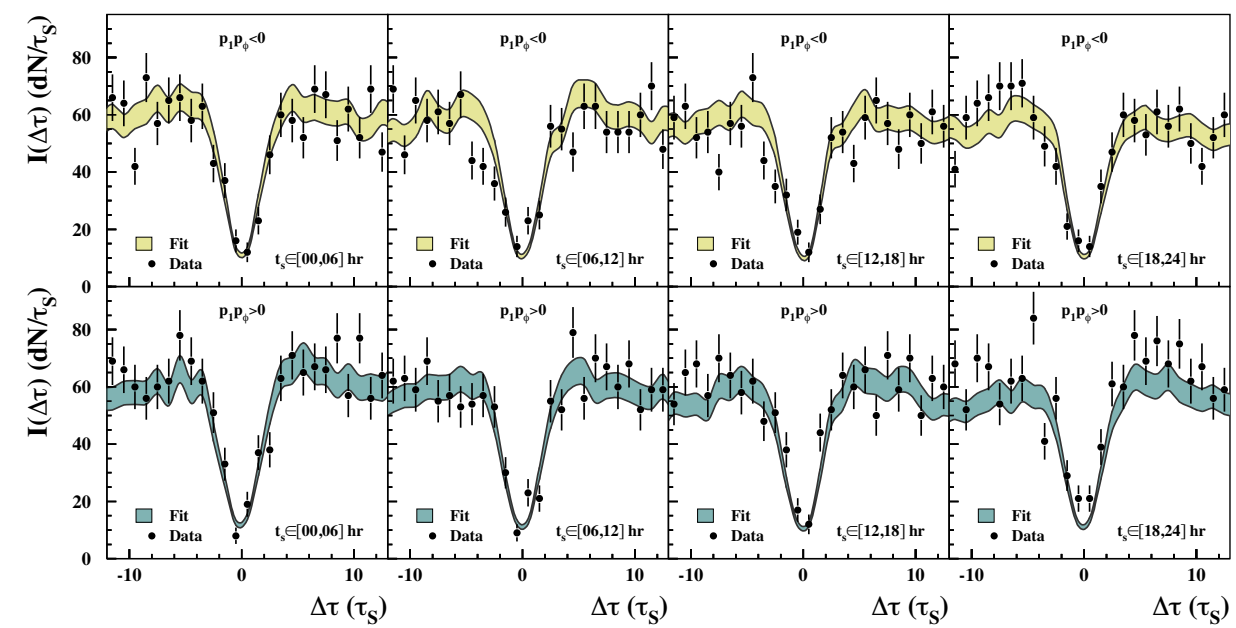

Figure 1. Fit to the distribution of the difference in proper time of neutral kaons as a function of sidereal time and particle direction in celestial coordinates [7].

The result is the measurement of all four CPT-violating parameters in the kaon sector of the SME [8]. In this context, $\Delta a_{\mu}$ parametrization of CPT-violation parameter is used:

$$
\delta_{k} \sim i \sin \phi_{\mathrm{Sw}} e^{i \phi_{\mathrm{SW}}} \gamma_{\mathrm{K}}\left(\Delta a_{0}-\overrightarrow{\beta_{\mathrm{K}}} \cdot \Delta \vec{a}\right) / \Delta m,
$$

where $\gamma_{\mathrm{K}}$ and $\overrightarrow{\beta_{\mathrm{K}}}$ are the kaon $\gamma$ factor and velocity in the laboratory frame, $\phi_{\mathrm{SW}}=\arctan (2 \Delta m / \Delta \Gamma)$ is the so-called superweak phase, $\Delta m=m_{\mathrm{L}}-m_{\mathrm{S}}, \Delta \Gamma=\Gamma_{\mathrm{S}}-\Gamma_{\mathrm{L}}$ are the mass and width differences for the neutral kaon mass eigenstates. The neutral kaon system is expressed by:

$$
\left|\mathrm{K}_{\mathrm{S}, \mathrm{L}}\right\rangle \propto\left(1+\epsilon_{S, L}\right)\left|\mathrm{K}^{0}\right\rangle \pm\left(1-\epsilon_{S, L}\right)\left|\overline{\mathrm{K}}^{0}\right\rangle, \quad \text { with } \epsilon_{\mathrm{S}, \mathrm{L}}=\epsilon_{\mathrm{K}} \pm \delta_{k} .
$$

Data reduction is based on two decay vertices with only two tracks each. For each vertex, invariant mass $\left|m_{\text {rec }}-m_{\mathrm{K}}\right|<5 \mathrm{MeV}$, missing mass $\sqrt{E_{\text {miss }}^{2}+\left|\vec{p}_{\text {miss }}\right|^{2}}<10 \mathrm{MeV},-50 \mathrm{MeV}^{2}<m_{\text {miss }}^{2}<10 \mathrm{MeV}^{2}$, and kaon momenta compatible with the 2-body decay hypothesis are required, the missing momentum being obtained from the analysis of Bhabha scattering events. A global likelihood function is built in order to kinematically constrain the event and to improve on the vertex resolution. 
Main background source is the kaon regeneration on the spherical beam pipe, suppressed by selecting events with both $K_{S}$ and $K_{L}$ decaying inside the beam pipe, that give a final contamination at the $2-3 \%$ level. Background contributions and analysis selection efficiencies are derived from MC simulation to which corrections from real data are applied. Full analysis chain was repeated several times varying all the cuts for the evaluation of the systematic uncertainties. The sum in quadrature of all the effects ranges between $30 \%$ and $40 \%$ of the statistical error. The results [7] are the most sensitive measurements in the quark sector:

$$
\begin{aligned}
& \Delta a_{o}=\left(-6.0 \pm 7.7_{\text {stat }} \pm 3.1_{\text {syst }}\right) \times 10^{-18} \mathrm{GeV} \\
& \Delta a_{x}=\left(0.9 \pm 1.5_{\text {stat }} \pm 0.6_{\text {syst }}\right) \times 10^{-18} \mathrm{GeV} \\
& \Delta a_{y}=\left(-2.0 \pm 1.5_{\text {stat }} \pm 0.5_{\text {syst }}\right) \times 10^{-18} \mathrm{GeV} \\
& \Delta a_{z}=\left(3.1 \pm 1.7_{\text {stat }} \pm 0.5_{\text {syst }}\right) \times 10^{-18} \mathrm{GeV}
\end{aligned}
$$

For comparison, the accuracy reached by similar measurements in B and D systems is of $O\left(10^{-13}\right) \mathrm{GeV}[8]$.

\section{The $\mathrm{K}^{+} \rightarrow \pi^{+} \pi^{-} \pi^{+}(\gamma)$ branching fraction}

The measurement of the branching fraction (BR) of $\mathrm{K}^{+} \rightarrow \pi^{+} \pi^{-} \pi^{+}(\gamma)$ completes the KLOE program of precision measurements of the dominant kaon branching fractions, fully inclusive of radiation effects [9-11]. It is based on an integrated luminosity of $\int \mathcal{L} \mathrm{dt} \simeq 174 \mathrm{pb}^{-1}$ that corresponds to $\sim 17$ million tagged $K^{+}$mesons. The availability of tagged kaons enables the precision measurement of absolute BRs providing the normalization sample. The analysis procedure consists of i) the selection of $\mathrm{K}^{+}$candidates (tagging procedure) by the identification of $\mathrm{K}^{-} \rightarrow \pi^{-} \pi^{0}$ and $\mathrm{K}^{-} \rightarrow \mu^{-} v$ samples, independently treated; ii) the reconstruction of the $\mathrm{K}^{+}$path from the kinematical constraints given by the $\mathrm{K}^{-}$momentum and $\phi$ momentum (from Bhabha-scattering events); iii) the backward extrapolation of any charged track not belonging to the $\mathrm{K}^{-}$decay chain; iv) the reconstruction of the $\mathrm{K}^{+}$decay vertex radial position $\left(\rho_{x y}\right)$ and the closest-approach distance $\left(\mathrm{CAd}_{i}\right)$ between the track and the $\mathrm{K}^{+}$ flight path; v) the selection of events with at least two tracks with $\operatorname{CAd}_{i} \leq 3 \mathrm{~cm}$ and $\rho_{x y} \leq 26 \mathrm{~cm}$, outside the drift chamber (DC) sensitive volume (for a better control of systematics from tagging procedure); vi) the measurement of the missing-mass distribution, $\mathrm{M}_{\text {miss }}^{2}=\left(\Delta \mathrm{E}_{K^{+}-\pi \pi}\right)^{2}-\left|\Delta \mathbf{P}_{K^{+}-\pi \pi}\right|^{2}$ (fig.2). The analysis is fully inclusive of radiative decays. The tagging procedure affects the selection efficiency of the $\mathrm{K}^{+} \rightarrow \pi^{+} \pi^{+} \pi^{-}$sample introducing a bias (tag-bias) on the BR measurement that has been evaluated from Monte Carlo simulation and used as a correction factor for the BR. Relating systematic error is estimated by changing the selection criteria of the tagging procedure. The other sources of systematics are the analysis cuts and the uncertainty on the kaon lifetime [13]. All of the contributions amount to $0.6 \%$ of relative systematic error [12]. In a sample of $12,065,087(5,171,239)$ $K^{-} \rightarrow \mu^{-} \bar{v}\left(K^{-} \rightarrow \pi^{-} \pi^{0}\right)$ tagging decays we found $N_{K \rightarrow 3 \pi}=48,032 \pm 286$ (20,063 \pm 186$)$ signal events, from which we derive fully consistent measurements of the absolute branching fractions:

$$
\begin{aligned}
& \left.B R\left(K^{+} \rightarrow \pi^{+} \pi^{-} \pi^{+}(\gamma)\right)\right|_{\operatorname{Tag}_{\mu 2}}=0.05552 \pm 0.00034_{\text {stat }} \pm 0.00034_{\text {syst }} \\
& \left.B R\left(K^{+} \rightarrow \pi^{+} \pi^{-} \pi^{+}(\gamma)\right)\right|_{T a g K_{\pi 2}}=0.05587 \pm 0.00053_{\text {stat }} \pm 0.00033_{\text {syst }} .
\end{aligned}
$$

The average, $B R\left(K^{+} \rightarrow \pi^{+} \pi^{-} \pi^{+}(\gamma)\right)=0.05565 \pm 0.00031_{\text {stat }} \pm 0.00025_{\text {syst }}$ [12] has a $0.72 \%$ accuracy, that is a factor $\sim 5$ better with respect to the previous measurement [14]. 


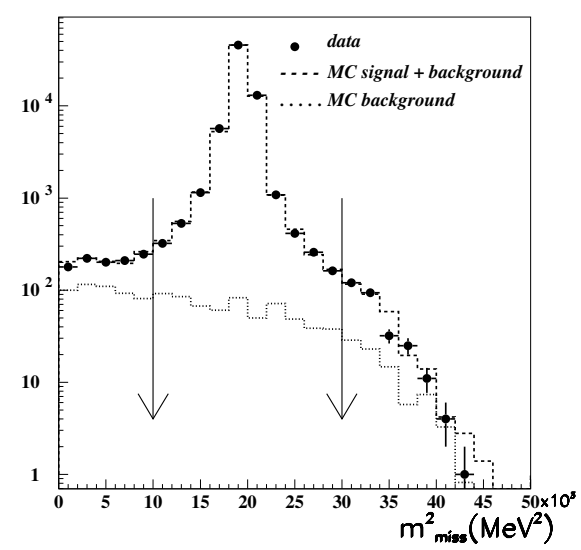

Figure 2. MC (dashed) and data (points) missing mass spectrum of the selected events. The arrows show the missing mass window for signal counting [12].

\section{Dark photon searches}

Some models of physics beyond the SM predict the existence of light neutral vector particles (the dark photon or U-boson) mediator of new gauge interactions under which ordinary matter is uncharged [15, 16]. Motivated by astrophysical arguments, their mass, $M_{U}$, is expected to be of order $1 \mathrm{GeV}$ or lighter [17, 18]. Dark photon coupling to SM could arise from kinetic mixing with the $\gamma / \mathrm{Z}$ boson [19] and regulated by a dimensionless parameter $\epsilon$ expected to be of $O\left(10^{-3}\right)$ or lower. These new particles can be observed as a sharp resonance at $M_{U}$ in the invariant mass distribution of charged lepton or pion pairs, in $e^{+} e^{-} \rightarrow l^{+} l^{-} \gamma$ or $V \rightarrow P l^{+} l^{-}$reactions, where $V(P)$ stands for any vector (pseudoscalar) meson, and $\mathrm{l}^{ \pm}$for muons, electrons or charged pions. KLOE has searched for $\mathrm{U}$ boson production using both, $\phi \rightarrow \eta e^{+} e^{-}$events (a), and $e^{+} e^{-} \rightarrow \mu^{+} \mu^{-} \gamma$ events (b). As for reaction (a), the papers of reference $[20,21]$ have been published in which the $\eta$ meson was identified from $\pi^{+} \pi^{-} \pi^{0}$ and $\pi^{0} \pi^{0} \pi^{0}$ decays in a sample of $1.7 \mathrm{fb}^{-1}$ of integrated luminosity at the $\phi$ peak. We obtained an excluded region at $90 \% \mathrm{CL}$ in the $M_{U}-\epsilon^{2}$ plane that rules out kinetic mixing above $810^{-6}$ in the mass range from 50-210 MeV (fig. 3). Reaction (b) was studied on the sample used for the measurement of the ratio $R=\sigma\left(e^{+} e^{-} \rightarrow \pi^{+} \pi^{-}\right) / \sigma\left(e^{+} e^{-} \rightarrow \mu^{+} \mu^{-}\right)$exploiting the precision MC simulation and data analysis chain of the QED process $e^{+} e^{-} \rightarrow \mu \mu \gamma$ published in reference [32].

A $\mu \mu \gamma$ candidate must have two tracks of opposite charge, with the point of closest approach to the $z$ axis within a cylinder of radius $8 \mathrm{~cm}$ and length $15 \mathrm{~cm}$ centered at the IR. We require two tracks at large polar angle, $50^{\circ}<\theta<130^{\circ}$, and one undetected photon. The separation between tracks and photon regions greatly reduces the contamination from both, the resonant $e^{+} e^{-} \rightarrow \phi \rightarrow \pi^{+} \pi^{-} \pi^{0}$, and FSR processes, $e^{+} e^{-} \rightarrow \pi^{+} \pi^{-} \gamma_{\mathrm{FSR}}$ and $e^{+} e^{-} \rightarrow \mu^{+} \mu^{-} \gamma_{\mathrm{FSR}}$. Electron, pion and muon pairs are identified by the mass obtained assuming an $e^{+} e^{-} \rightarrow x^{+} x^{-} \gamma$ process. In addition, a pseudo-likelihood estimator based on time-of-flight and calorimeter information is used for improving on the separation of electrons from pions and muons. Residual contamination and analysis efficiency is evaluated from MC simulations that have been controlled with real data samples. The differential $\mu \mu \gamma$ cross section is in excellent agreement with the expectations from the NLO PHOKHARA generator [33]. The exclusion plot for $e^{+} e^{-} \rightarrow \mathrm{U} \gamma \rightarrow \mu^{+} \mu^{-} \gamma$ is obtained using the $\mathrm{CL}_{\mathrm{S}}$ technique. It covers the mass region $520<M_{U}<980 \mathrm{MeV}$ (fig. 3), with upper limits at 90\% CL on $\epsilon^{2}$ from $8.610^{-7}$ to $1.610^{-5}$. 


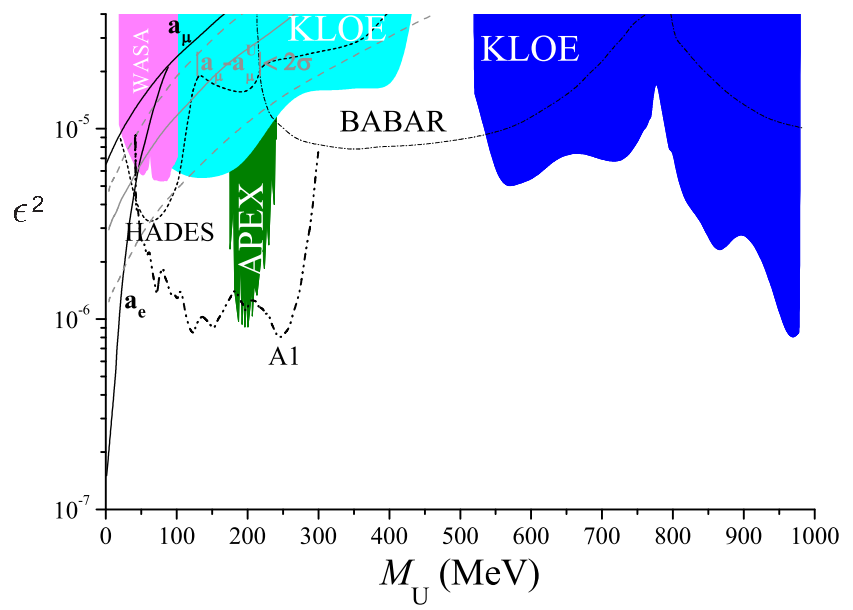

Figure 3. Exclusion plot at $90 \% \mathrm{CL}$ in the $\mathrm{M}_{U}-\epsilon^{2}$ plane obtained from the analysis of $\phi \rightarrow \eta e^{+} e^{-}$(cyan) and the $e^{+} e^{-} \rightarrow \mu^{+} \mu^{-} \gamma$ (blue) final states [22]. Current limits from the A1 (dash-double dotted [23, 24]), Apex (green [25]), WASA (magenta [26]), and HADES (dashed [27]) experiments are also shown. The dash-dotted line is an estimate using BaBar data [28-30]. The solid lines are the limits from muon and electron anomaly [31]. Gray line shows values that could explain the $a_{\mu}$ discrepancy.

\section{Conclusions}

KLOE recently obtained several results on kaon and hadron physics, and performed dark photon searches in the mass range from $20-980 \mathrm{MeV}$. Further studies on the interference pattern of neutral kaon pairs are in progress to improve on the sensitivity of quantum-mechanics tests with entangled meson systems. As for the dark photon searches, the ongoing analysis of the $e^{+} e^{-} \rightarrow \mathrm{U} \gamma \rightarrow e^{+} e^{-} \gamma$ process will extend the sensitivity to low masses, in the $2-200 \mathrm{MeV}$ range, and the studies of the final state with two muons plus missing energy are investigating dark photon production through darkhiggsstrahlung, $e^{+} e^{-} \rightarrow \mathrm{Uh}$, assuming $\mathrm{m}_{h^{\prime}}<\mathrm{m}_{U}$. In order to extend the KLOE physics program [6], the innermost part of the detector has been upgraded including tagger stations for $\gamma-\gamma$ physics, an inner tracker to improve on vertex resolution near the IR, and calorimeters both, to increase the acceptance at low polar angle, and to instrument the $D A \Phi N E$ focusing region.

\section{References}

[1] M. Adinolfi et al., Nucl. Instrum. Meth. A 488, 51 (2002).

[2] M. Adinolfi et al., Nucl. Instrum. Meth. A 482, 364 (2002).

[3] A. Balla et al., Acta Phys. Polon. Supp. 6, 1053 (2013).

[4] F. Happacher et al., Nucl. Phys. Proc. Suppl. 197, 215 (2009).

[5] M. Cordelli, G. Corradi, F. Happacher et al., Nucl. Instrum. Meth. A 617, 105 (2010).

[6] G. Amelino-Camelia, F. Archilli, D. Babusci et al., Eur. Phys. J. C 68, 619 (2010).

[7] D. Babusci et al. (KLOE-2), Phys. Lett. B 730, 89 (2014).

[8] V.A. Kostelecky, N. Russell, Rev. Mod. Phys. 83, 11 (2011).

[9] F. Ambrosino et al. (KLOE), Phys. Lett. B 632, 76 (2006).

[10] F. Ambrosino et al. (KLOE), J. High Energy Phys. 02, 098 (2008). 
[11] A. Aloisio et al. (KLOE), Phys. Lett. B 597, 139 (2004).

[12] D. Babusci et al. (KLOE/KLOE-2), Phys. Lett. B 738, 128 (2014).

[13] F. Ambrosino et al. (KLOE), J. High Energy Phys. 01, 073 (2008).

[14] I. Chiang et al., Phys. Rev. D 6, 1254 (1972).

[15] P. Fayet, Phys. Lett. B 95, 285 (1980).

[16] B. Batell, M. Pospelov, A. Ritz, Phys. Rev. D 80, 095024 (2009).

[17] N. Arkani-Hamed, N. Weiner, J. High. Energy Phys. 12, 104 (2008).

[18] M. Pospelov, A. Ritz, M.B. Voloshin, Phys. Lett. B 662, 53 (2008).

[19] B. Holdom, Phys. Lett. B 166, 196 (1986).

[20] F. Archilli et al. (KLOE-2), Phys. Lett. B 706, 251 (2012).

[21] D. Babusci et al. (KLOE-2), Phys. Lett. B 720, 111 (2013).

[22] D. Babusci et al. (KLOE-2), Phys. Lett. B 736, 459 (2014).

[23] H. Merkel et al. (A1), Phys. Rev. Lett. 106, 251802 (2011).

[24] H. Merkel et al., Phys. Rev. Lett. 112, 221802 (2014).

[25] S. Abrahamyan et al. (APEX), Phys. Rev. Lett. 107, 191804 (2011).

[26] P. Adlarson et al. (WASA-at-COSY), Phys. Lett. B 726, 187 (2013).

[27] G. Agakishiev et al. (HADES), Phys. Lett. B 731, 265 (2014).

[28] M. Reece, L.T. Wang, J. High Energy Phys. 07, 051 (2009).

[29] J.D. Bjorken, R. Essig, P. Schuster, N. Toro, Phys. Rev. D 80, 075018 (2009).

[30] B. Aubert et al. (BaBar), Phys. Rev. Lett. 103, 081803 (2009).

[31] M. Pospelov, Phys. Rev. D 80, 095002 (2009).

[32] D. Babusci et al. (KLOE), Phys. Lett. B 720, 336 (2013).

[33] H. Czyz, A. Grzelinska, J.H. Kuhn, G. Rodrigo, Eur. Phys. J. C 39, 411 (2005). 